INPLASY

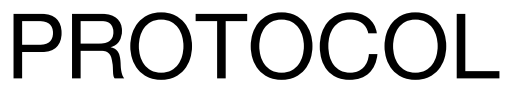

To cite: Zhang et al. The application of stem cells in tissue engineering for periodontal defects in randomized controlled trial: a systematic review and metaanalysis. Inplasy protocol 202210036. doi: 10.37766/inplasy2022.1.0036

Received: 08 January 2022

Published: 08 January 2022

Corresponding author: Qiyan Li

ynliqiyan@aliyun.com

Author Affiliation: Department of Stomatology, The First People's Hospital of Yunnan Province, Kunming, 650032, Yunnan, China.

Support: None.

Review Stage at time of this submission: Preliminary searches.

Conflicts of interest:

None declared.

\section{The application of stem cells in tissue engineering for periodontal defects in randomized controlled trial: a systematic review and meta-analysis}

\author{
Zhang, YH¹; Zhao, WH²; Jia, LY3; Xu, N4; Xiao, Y5; Li, QY6.
}

Review question / Objective: P:Moderate to severe chronic periodontitis patients (1) with probing depth (PD) $\geq 5 \mathrm{~mm}$, clinical attachment level (CAL) $\geq 5 \mathrm{~mm}$, bone defect depth (BDD) $\geq 3 \mathrm{~mm}$, and plaque index $<30 \%$. l:tissue engineering treatment using stem cells applied to the experimental group. C:conventional periodontal regeneration therapy applied to the control group. O:probing depth(PD), clinical attachment level(CAL), bone defect depth(BDD), and gingival recession (GR),Or the change in these indices. S:The PubMed, Cochrane Library, Embase, SinoMed, CNKI and Wanfang databases were searched for clinical studies related to the application of stem cells in tissue engineering in the treatment of periodontal tissue defects, According to certain inclusion and exclusion criteria, obtain as many clinical RCT studies as possible for Meta analysis and systematically evaluate the practical application of stem cell tissue engineering in human periodontal regeneration.

INPLASY registration number: This protocol was registered with the International Platform of Registered Systematic Review and Meta-Analysis Protocols (INPLASY) on 08 January 2022 and was last updated on 08 January 2022 (registration number INPLASY202210036).

\section{INTRODUCTION}

Review question / Objective: P:Moderate to severe chronic periodontitis patients (1) with probing depth (PD) $\geq 5 \mathrm{~mm}$, clinical attachment level (CAL) $\geq 5 \mathrm{~mm}$, bone defect depth (BDD) $\geq 3 \mathrm{~mm}$, and plaque index $<30 \%$. I:tissue engineering treatment using stem cells applied to the experimental group. C:conventional periodontal regeneration therapy applied to the control group. O:probing depth(PD), clinical attachment level(CAL), bone defect depth(BDD), and gingival recession (GR),Or 
the change in these indices. S:The PubMed, Cochrane Library, Embase, SinoMed, CNKI and Wanfang databases were searched for clinical studies related to the application of stem cells in tissue engineering in the treatment of periodontal tissue defects, According to certain inclusion and exclusion criteria, obtain as many clinical RCT studies as possible for Meta analysis and systematically evaluate the practical application of stem cell tissue engineering in human periodontal regeneration.

Rationale: Periodontitis is an infectious disease characterized by bacteria-induced chronic inflammation and caused by multiple factors, leading to progressive and irreversible destruction of the periodontal attachment apparatus. When periodontitis symptoms are detected, patients already have extensive periodontal tissue defects, such as periodontal pocket formation, clinical attachment loose, gingival recession, and tooth loss.Traditional treatments for periodontal defects, such as supragingival cleaning, subgingival curettage, and root surface leveling, have difficulty restoring periodontal tissues. New clinical periodontal regenerative therapies, such as guided tissue regeneration (GTR), bone grafts, growth factors, and biological scaffold materials alone or in combination, can improve the degree of periodontal defects. However, these methods have limitations with respect to the complete regeneration of periodontal tissue and are unable to repair severe periodontitis lesions in the end stage. With the grafting of therapeutic cells and/or growth factors into biodegradable scaffolds, tissue engineering provides a new solution for periodontal tissue regeneration.Numerous animal experiments and case reports have confirmed the significant advantages of stem cells in periodontal tissue regeneration.

Condition being studied: Although clinical trials have examined the application of stem cells in periodontal regeneration and showed good results, systematic literature review is not much. Moreover, quantitative evaluations of the application of stem cells in randomized controlled trials (RCTs) are lacking. Novello et al. conducted metaanalysis on the clinical application of Mesenchymal stem cells in periodontal regeneration, only 2 RCT studies were included, the potential heterogeneity and uncertainty might lead to unreliable results. Therefore, this study intends to obtain as many clinical RCT studies as possible for Meta analysis and systematically evaluate the practical application of stem cell tissue engineering in human periodontal regeneration.

\section{METHODS}

Search strategy: The PubMed, Cochrane Library, Embase, SinoMed, CNKI and Wanfang databases were searched, the first three are English databases and the last three are Chinese databases. The retrieval time was from the establishment of the corresponding database to May 9, 2021. A combination of subject headings and free-text terms was applied. The search terms included periodontitis, chronic periodontitis, periodontitis tissue regeneration, alveolar bone repair, periodontal regenerative treatment, periodontal tissue engineering, odontogenic stem cells, oral stem cells, periodontal stem cells, and clinical application of oral stem cells in both the English and Chinese databases. In addition, the references of the included articles were reviewed to supplement relevant literature.

Participant or population: Moderate to severe chronic periodontitis patients.

Intervention: Tissue engineering treatment using stem cells applied to the experimental group.

Comparator: Conventional periodontal regeneration therapy applied to the control group.

Study designs to be included: Only randomized controlled trial.

Eligibility criteria: Eligibility criteria including:(1) with probing depth (PD) $\geq 5$ $\mathrm{mm}$, clinical attachment level $(\mathrm{CAL}) \geq 5$ 
$\mathrm{mm}$, bone defect depth (BDD) $\geq 3 \mathrm{~mm}$, and plaque index < 30\%; (2) over 18 years of age with no limitations on gender, race and nationality; (3) with no contraindications, complications or systemic diseases; (4) with professional periodontal basic treatment received before therapy, including supragingival cleaning, subgingival scraping, root surface leveling, oral hygiene guidance, etc.; (5) with no tobacco and alcohol intake; (6) not pregnant and lactating; (7) with no antiinflammatory drugs used before treatment and no other periodontal surgery used to treat the affected teeth; and (8) with no bone metabolic diseases but good treatment compliance. Exclusion criteria: (1) Reviews, treatises or conference papers; (2) in vitro experiments or animal experiments; (3) non-RCTs; (4) Follow-up was less than three months; (5) lack of data or lack of access to the full text; (6) duplicate publications or data; and (7) Nonchinese and English literature.

Information sources: The PubMed, Cochrane Library, Embase, SinoMed, CNKI and Wanfang databases were searched for clinical studies related to the application of stem cells in tissue engineering in the treatment of periodontal tissue defects.The retrieval time was from the establishment of the corresponding database to May 9, 2021. Two reviewers independently read the titles, abstracts and full texts of the retrieved articles; screened the articles strictly according to the inclusion and exclusion criteria; and collected and crosschecked the results.In case of disagreement, a third reviewer was consulted to assist in judgment. The authors of the included studies were contacted if any missing information was needed. Does not include unpublished grey literature.

Main outcome(s): Probing depth(PD), clinical attachment level(CAL), bone defect depth(BDD), and gingival recession (GR), Or the change in these indices.

Data management: The following data were screened: (1) basic information, including the title, first author, date and journal of publication, and experimental period; (2) basic characteristics of subjects, including age, sex, sample size, symptoms, and interventions; (3) data to assess risk of bias; and (4) results (mean \pm standard deviation). Extract and record using Excel.

Quality assessment / Risk of bias analysis: The risk of bias in the RCTs was independently evaluated and crosschecked by two investigators. The risk assessment tools employed were those recommended by the Cochrane Manual, and the following features were assessed: (1) the presence of a correct specific random sequence, (2) the use of allocation concealment, (3) the blinding of researchers and subjects, (4) the evaluation of results using a blind method, (5) complete data (avoidance of potential loss of follow-up), (6) the absence of selective reporting of research results, and (7) bias from other sources.

Strategy of data synthesis: The mean difference (MD), point estimates and 95\% CI were calculated for continuous variables. The $Q$ statistic test and 12 test were used to evaluate the heterogeneity of the parameters. If $P>0.1$ and $I 2<50 \%$, the included studies were considered to be homogenous, and meta-analysis was performed using a fixed-effect model. If $\mathbf{P} \leq$ 0.1 and $12 \geq 50 \%$, heterogeneity was considered present, and a random-effects model was used for the metaanalysis.Sensitivity analysis and subgroup analysis were performed to analyze the sources of heterogeneity for the combined results with significant heterogeneity. RevMan 5.4 software was used for this meta-analysis.

Subgroup analysis: Subgroup analysis was performed on the main output results, including probing depth(PD), clinical attachment level(CAL), bone defect depth(BDD), and gingival recession (GR). It is planned to conduct subgroup analysis on the follow-up time, stem cell type and biological scaffold type of the included RCTs studies. There may not be many studies included, resulting in insufficient 
studies in the subgroup and a lack of interpretation of the results.

Sensitivity analysis: One by one exclusion study, subgroup analysis or alternative outcome variables.

Language: Only Chinese and English literature are included.

Country(ies) involved: China.

Other relevant information: None.

Keywords: Tissue engineering; Stem cells; Periodontal defect; Periodontium regeneration; Randomized controlled trial.

Contributions of each author:

Author 1 - Yuhao Zhang - Data curation, Formal analysis, and Writing- Original draft preparation.

Email: limonhao@163.com

Author 2 - Wenheng Zhao - Methodology, Investigation, and Writing- Reviewing and Editing.

Email: zwh787506105@163.com

Author 3 - Liyang Jia - Visualization, Investigation.

Email: edward930108@163.com

Author 4 - Nan Xu - Resources, Project administration.

Email: 872094825@qq.com

Author 5 - Yan Xiao - Validation.

Email: 2314060662@qq.com

Author 6 - Qiyan Li - Conceptualization, Funding acquisition, and Supervision.

Email: ynliqiyan@aliyun.com 